\title{
Absolute configuration correlation studies in solid state organic photochemistry
}

\author{
Mordechai Leibovitch, Gunnar Olovsson, John R. Scheffer* and James Trotter \\ Department of Chemistry, University of British Columbia, 2036 Main Mall \\ Vancouver, British Columbia, Canada V6T 1Z1
}

\begin{abstract}
An ever-increasing number of examples in the literature attest to the fact that photochemical reactions carried out in optically active crystalline media can lead to enantiomerically enriched products in high optical yields. Taken from our own work and that of others, this article examines the relatively few examples of such reactions in which the absolute stereochemical arrangements of the molecules in the reactant crystals are correlated with the absolute configurations of the optically active products formed by irradiation of the same crystals. Studies of this type provide penetrating insights into the mechanisms of the reactions in question, and in addition, help to pinpoint the crystal lattice-derived stereoelectronic factors that are responsible for the observed enantioselectivities.
\end{abstract}

\section{INTRODUCTION}

In isotropic media, photochemical reactions that transform achiral reactants into chiral products lead to racemic mixtures. When such reactions are conducted in optically active media, however, the previously enantiomeric transition states become diastereomerically related owing to their interaction with the medium. These transition states will, in general, have unequal energies, the result being that the formation of one photoproduct enantiomer will be favored kinetically over the other. In other words, an asymmetric induction will occur. In media where the interaction with the reactant is relatively weak and transitory, as in the case of optically active solvents, photoproduct enantiomeric excesses tend to be low (ref. 1). On the other hand, it is now well established that when photoreactions of this type are conducted in optically active crystalline media, where the intermolecular forces are stronger and much more highly organized, the extent of asymmetric induction can be quite high (ref. 2).

It is beyond the scope of the present article to review all cases of solid state asymmetric induction. Instead, we concentrate here on the few published examples of reactions of this type in which the absolute structure of the reactants is correlated with the absolute configuration of the optically active products. Whether in solution (for example, the $S_{\mathrm{N}} 2$ reaction) or in the crystalline state (as in the present instance), such studies represent one of the most powerful methods at the disposal of the organic chemist for elucidating reaction mechanisms. In addition, for reactions in the solid state, absolute configuration correlations tell us a great deal about the crystal lattice forces that govern excited state behavior-information that can be used to advantage in designing new crystalline materials to fulfill specific functions in chemistry and materials science, that is, in the burgeoning field of "crystal engineering."

\section{ABSOLUTE CONFIGURATION CORRELATIONS}

\section{Photoaddition Reactions between Ketones and Deoxycholic Acid}

One of the first applications of the absolute configuration correlation method to an enantioselective photochemical reaction in the crystalline state was carried out in 1980 by Lahav, Leiserowitz et al. (ref. 3). These authors reported that acetophenone forms a 2:5 crystalline channel inclusion complex with deoxycholic acid (1) as the host, and that irradiation of the complex in the solid state Jeads to abstraction 
by the acetophenone of a hydrogen atom from $\mathrm{C} 5$ of the steroid followed by coupling of the resulting radical pair to produce photoadduct 2 (Scheme 1). Because the crystals retained their singularity throughout the photolysis, (a so-called single crystal-to-single crystal or topotactic transformation), the course of the reaction (including intermediate stages) could be followed by $\mathrm{X}$-ray crystallography. In this case, the crystals are optically active by virtue of the optical activity of the deoxycholic acid, and since the absolute configuration of this compound is known, the crystal structures establish the absolute orientation of the reacting deoxycholic acid-acetophenone pair as well as the absolute configuration of the newly formed stereogenic center in photoproduct 2 , which is (S).

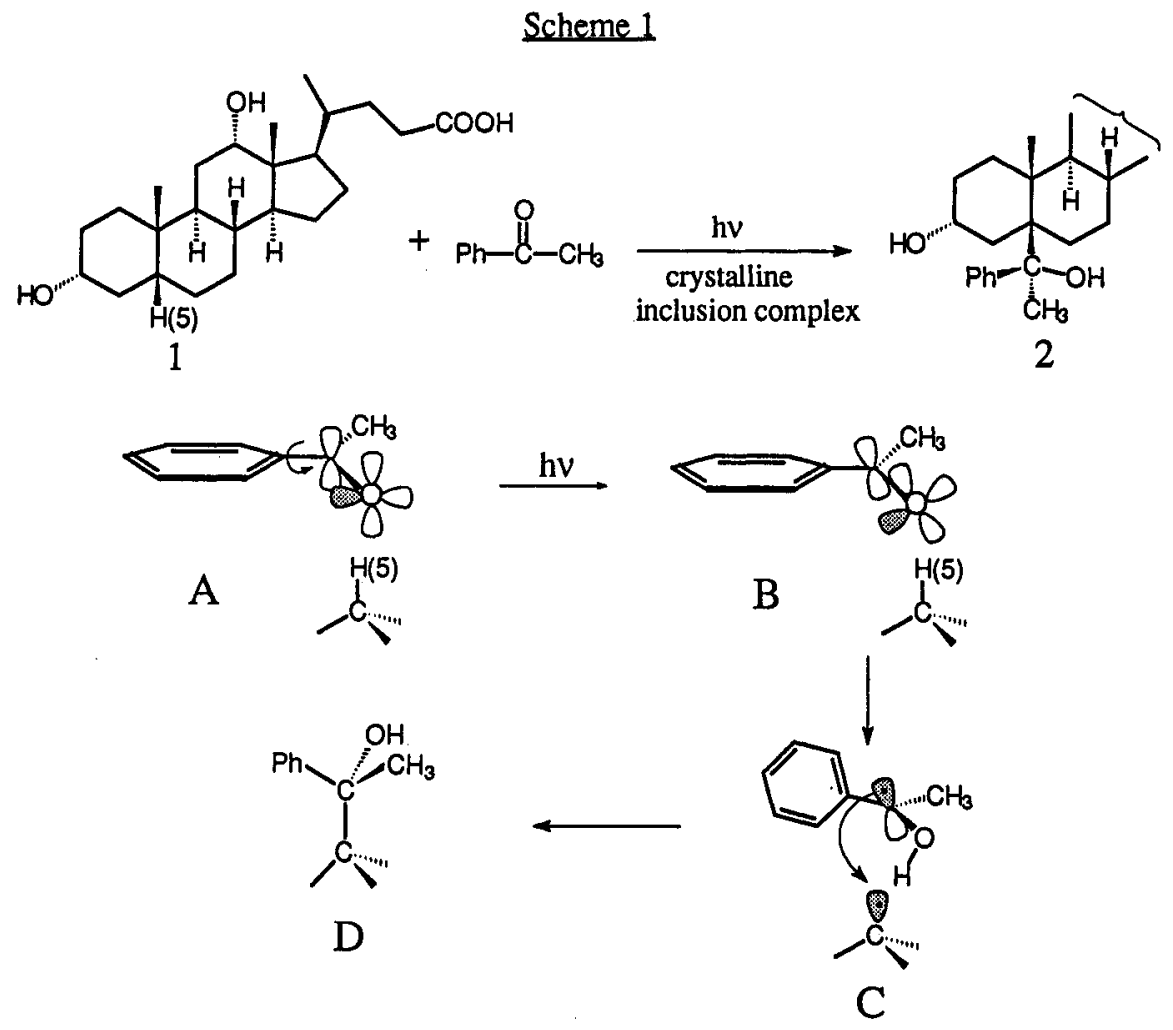

The absolute configuration correlation revealed a surprising result, namely that photoproduct 2 has a configuration opposite to that expected on the basis of the host-guest packing at the reaction site (structure A, Scheme 1). This finding means that there had to have been a net $180^{\circ}$ rotation of the acetyl group at some point along the reaction coordinate, and the authors suggested that this motion is initiated by the need for the oxygen n-orbital to rotate into position to abstract $\mathrm{H} 5$ of the steroid (structure $\mathrm{B}$ ). Following abstraction (structure C), the odd electron-containing orbital at C5 is closer to the top lobe of the p-orbital on what was originally the carbonyl carbon, and radical coupling thus results in an overall inversion of configuration at this center (structure D) relative to the original arrangement in structure $A$. Allowing for minor adjustments in the positions of the neighboring steroid molecules, atom-atom potential energy calculations revealed that the postulated $180^{\circ}$ rotation can in fact take place without incurring prohibitive intermolecular contacts within the crystal lattice.

Similar photochemical and crystallographic studies were carried out on the complexes of deoxycholic acid with $p$-fluoroacetophenone and propiophenone (ref. 3). p-Fluoroacetophenone was found to behave similarly to acetophenone, but in the case of propiophenone, different results were obtained. Here, in spite of a packing arrangement in the crystal similar to that found for acetophenone, both diastereomeric photoaddition products at C5 were obtained in an unspecified ratio, one having the (S) absolute configuration at the newly created stereogenic center and the other $(R)$. The authors rationalized these results on the basis of the relative size of ethyl versus methyl, suggesting that the larger ethyl group experiences more resistance from the crystalline medium to the $180^{\circ}$ rotation required for formation of the (S) photoproduct, thus allowing formation of the $(R)$ product to compete. 


\section{The Di- $\pi$-Methane Photorearrangement of Diisopropyl 2.10-Dihydro-2.10-ethenoanthracene-} 11.12-Dicarboxylate

In this section we discuss work from our own laboratory on diisopropyl 9,10-dihydro-9,10ethenoanthracene-11,12-dicarboxylate (3, Scheme 2) (ref. 4). This compound, which has average $C_{2 v}$ symmetry in solution, adopts a chiral conformation in the solid state and crystallizes in the chiral space group $\mathrm{P} 22_{1} 2_{1}$. Crystals that have chiral space groups are characterized as being enantiomorphous, that is, they exist in "right-handed" and "left-handed" forms that may or may not be visually distinguishable. Crystals grown from optically active materials (such as the deoxycholic acid-ketone complexes) will be either all right-handed or all left-handed depending on which enantiomer of the chiral component is employed. In the case of chiral crystals grown from achiral substances, however, it is not uncommon to find both enantiomorphs present in a given batch of crystals from the same recrystallization. In order to ensure that one is working with an "optically pure" crystal, therefore, an X-ray quality single crystal should be chosen. Alternatively, polycrystalline samples of the same handedness may be prepared by seeding techniques using seeds obtained by crushing single crystals.

Irradiation of a single crystal of diester 3 led to compound 4 as the sole product, an example of the well known di- $\pi$-methane rearrangement reaction (ref. 5). Photoproduct 4 is chiral, and when its optical purity was determined, it proved to be nearly optically pure ( $>95 \%$ enantiomeric excess). The process was shown to be fully enantioselective, since photolysis of one enantiomorphous single crystal led to one enantiomer of 4, while irradiation of the opposite enantiomorph led to the other. Reactions such as this, in which an achiral reactant is transformed into an enantiomerically enriched product without the external intervention of pre-existing optical activity, have been termed absolute asymmetric syntheses. This is a somewhat confusing use of the adjective absolute, since this term is more commonly used in stereochemistry to refer to absolute configuration, which is the sense in which we shall be using it in this article.
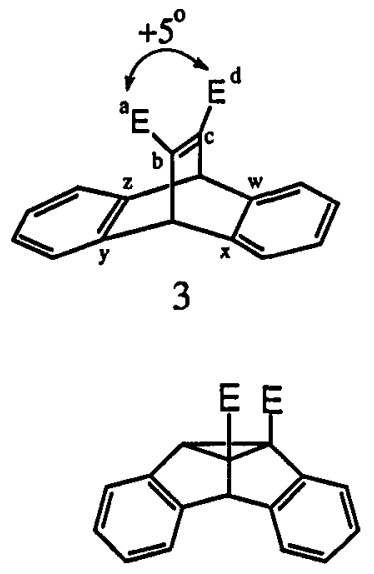

$(\mathbf{S}, \mathbf{S}, \mathbf{S}, \mathbf{S})-(-)-4$

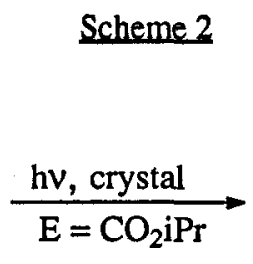

Path I: c...w

Path II: b...y

Path III: b...x

Path IV: c...z
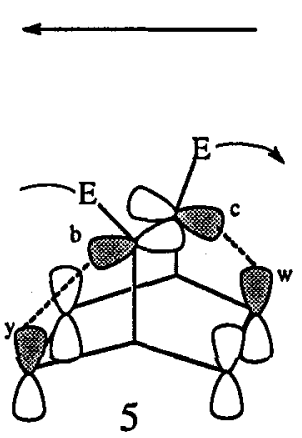
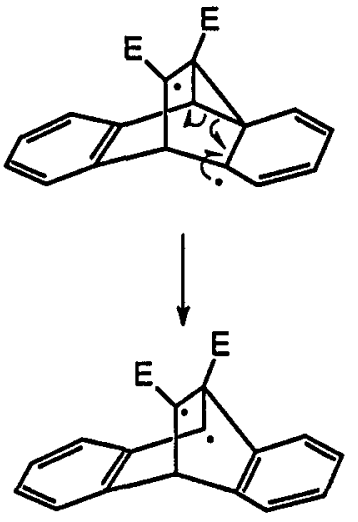<smiles>CCC</smiles> 
differentiate between the two possibilities, and it was to solve this problem that we tumed to the absolute configuration correlation studies.

Because the photoreaction of diester 3 is not single crystal-to-single crystal in nature, it was necessary to carry out the absolute configuration correlation in several steps. The first step was to divide a large single crystal of diester 3 into two fragments. One fragment was subjected to anomalous dispersion Xray crystallography (Bijvoet method, ref. 6). As mentioned above, diester 3 adopts a chiral conformation in the solid state, and one way of denoting its absolute configuration is by specifying the sign of the dihedral angle between the carbonyl carbons of the ester groups and the bridging double bond (dihedral angle $a-b-c-d$, Scheme 2). This angle is non-zero owing to steric interference between the bulky ester groups, and in the crystal fragment chosen, its value was $+5^{\circ}$. At the same time, the second crystal fragment was photolyzed and the resulting optically active product 4 recrystallized and subjected once again to Bijvoet analysis. This showed that the photoproduct had the all-(S) absolute configuration. Scheme 2 depicts the correct absolute configurations for both reactant and photoproduct as deduced crystallographically.

These results establish unambiguously that the solid state photoreaction of diester 3 proceeds through pathways I and/or II, since paths III and/or IV would have given the all (R) enantiomer of 4 . The question then arises, why are pathways I and/or II favored over III and/or IV? There appear to be two reasonable answers to this question. First, because of the positive dihedral angle $a-b-c-d$, the orbital at position $c$ is tilted toward benzo position $w$, and the orbital at $b$ is tilted toward $y$. In other words, there is better orbital overlap for benzo-vinyl bridging of type I and II than for type III and IV. This is depicted in structure 5, Scheme 2. Secondly, pathways I and II lead to a diminution of the steric interaction between the bulky ester groups. In the alternative pathways leading to the unobserved enantiomer, the ester substituents would be driven toward each other during benzo-vinyl bridging. As we shall see, similar arguments successfully account for the absolute steric courses of several other solid state di- $\pi$ methane photorearrangements.

A final point concerns the interesting question of whether it is possible, with the data in hand, to determine the relative importance of paths I and II. While the absolute configuration correlations do not help in this regard, the packing diagram for diester 3 indicates that the ester substituent $d$, which is attached to the vinyl carbon involved in path $I$, is in a much more congested steric environment than its path II counterpart. Because the ester substituent attached to the reactive site is required to move most during the initial stages of the rearrangement, there is a clear prediction of a preference for path II. This qualitative conclusion was borne out quantitatively by atom-atom potential energy calculations similar to those mentioned above, which simulate the steric interactions developed between the reactant and its lattice neighbors during the early stages of rearrangement.

\section{The Photochemistry of Inclusion Complexes of 9,10-Dihydro-9,10-ethenoanthracene-11,12-" bis(diphenylphosphine oxide)}

Because Bijvoet absolute configuration assignments for molecules that lack heavy atoms (such as 3 and 4, above) are subject to some uncertainty, and because we wished to know whether the structurereactivity relationships developed above work for other systems, we sought additional ethenoanthracene derivatives for study. Serendipitously, 9,10-dihydro-9,10-ethenoanthracene-11,12bis(diphenylphosphine oxide) (6, Scheme 3) was found to form a 1:1 inclusion complex with ethanol that crystallized in the chiral space group $\mathrm{P}_{1} 2_{1} 2_{1}$ (ref. 7). Compound 6 has the advantage that it contains phosphorous, a sufficiently heavy atom to allow unambiguous assignment of absolute configuration by the Bijvoet method.

Like its counterpart 3, adduct 6 was found to undergo the di- $\pi$-methane photorearrangement in solution as well as in the solid state. The solution photoproduct, 7, was, as expected, racemic, but irradiation of single crystals of the ethanol adduct of 6 led to photoproduct 7 in optically active form (89\% ee at $84 \%$ conversion, room temperature). Even higher ees could be achieved at lower conversions and/or lower photolysis temperatures. As before, the use of enantiomorphous single crystals led to the optical antipodes of 7 . 
By chance, the single crystal of complex $6 \bullet \mathrm{EtOH}$ chosen for Bijvoet analysis proved to have the same absolute configuration as the crystal of diester 3 that was studied. In the case of 6 , the P-C=C-P dihedral angle was $+23.9^{\circ}$, much larger than the corresponding angle for 3 , a finding that can be ascribed to the bulkier nature of the two diphenylphosphine oxide substituents. Since crystals of photoproduct 7 were not suitable for $\mathrm{X}$-ray diffraction, the absolute configuration of this compound was determined from a crystal structure of the corresponding bis(phosphine) prepared by trichlorosilane reduction of optically pure 7. This showed that the reduction product (and hence photoproduct 7 ) formed by photolysis of (t)6 had the all-(S) absolute configuration. This is exactly the same correlation as found in the case of diester 3, a result that can be taken as strong support for the mechanistic analysis presented above in which pathways I and/or II are favored by a combination of steric and orbital overlap factors. Further support for this picture comes from unpublished work with the corresponding complexes of 6 with 1propanol and 2-propanol (ref. 8). Both complexes crystallize in chiral space groups $\left(\mathrm{P}_{2} 2_{1} 2_{1}\right)$, and photolysis of single crystals of these materials give compound 7 in $>90 \% e e$. In both cases, the absolute configuration assignments between reactant and photoproduct were made successfully, and the results conformed to the mechanistic picture outlined above. Reassuringly, in the case of the 1-propanol and 2propanol complexes, the crystals chosen for analysis had negative $\mathrm{P}-\mathrm{C}=\mathrm{C}-\mathrm{P}$ dihedral angles $\left(-23^{\circ}\right.$ and $21^{\circ}$, respectively) and led to the all-(R) enantiomer of photoproduct 7 . Also in accord with this picture was the finding that the ethyl acetate complex of 6 , which crystallizes in the achiral space group $P 2_{1} / \mathrm{c}$ and therefore contains equal amounts of $(+)$ - and (-)-conformers, leads to racemic 7 when irradiated in the solid state.

\section{Ionic Chiral Auxiliary-Induced Solid State Asymmetric Synthesis}

In the previous two examples involving ethenoanthracene derivatives 3 and 6 , the chiral space group necessary for asymmetric induction in the crystalline state was produced spontaneously. That is, the compounds had an inherent and natural tendency to crystallize in this manner. Such circumstances are rare, however, and most compounds like 3 and $\mathbf{6}$ that are achiral in solution prefer to crystallize in space groups that are also achiral (ref. 9). As a result, spontaneous crystallization of achiral compounds in chiral space groups cannot be relied upon as a general technique leading to asymmetric synthesis, and a more reliable method is required. To accomplish this, we have introduced the technique of the ionic chiral auxiliary (ref. 10). Consider, for example, the photochemical reaction of an achiral carboxylic acid that leads to a chiral product. If we treat the carboxylic acid with an optically active amine and then photolyze the resulting salt crystals (which are required to be in a chiral space group), two diastereomeric photoproduct salts can be formed. If one of these is formed in greater amounts than the other, an asymmetric induction has been achieved and the optically active ammonium ion has acted as an ionic chiral auxiliary. Of course the opposite approach, in which the ammonium ion is the prochiral photoreactant and the carboxylate anion is the ionic chiral auxiliary, is equally feasible. In this section and the one that follows we provide examples of this method of asymmetric synthesis and show how absolute configuration correlations between reactant and photoproduct provide insight into the details of the reactions involved.

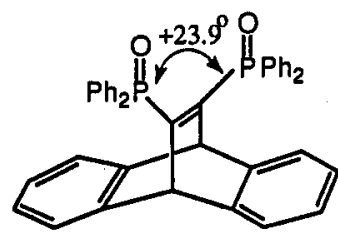

$6 \cdot \mathrm{EtOH}$

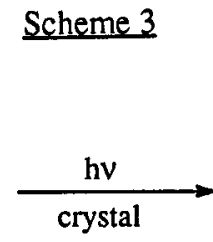

crystal

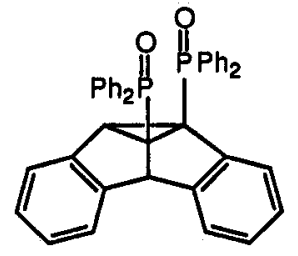

7

The first example we shall discuss involves carboxylic acid 8 (Scheme 4), another di- $\pi$-methane photoreactant. Treatment of this compound with $(S, S)-(+)$-pseudoephedrine (9) afforded salt 10, and irradiation of crystals of this material, followed by acidic workup and treatment with diazomethane, gave methyl ester 11 (ref. 11). The regioselectivity of this reaction is in accord with expectations based on the relative radical stabilizing and polar effects of the vinyl substituents on the initially formed biradical (ref. 12). NMR spectroscopy using Eu(hfc) ${ }_{3}$ as a chiral shift reagent showed that photoproduct 11 was formed in over $95 \%$ ee, $(+)$ enantiomer predominating; use of $(R, R)-(-)$-pseudoephedrine led to the $(-)$ enantiomer with the same ee. 


\section{Scheme 4}

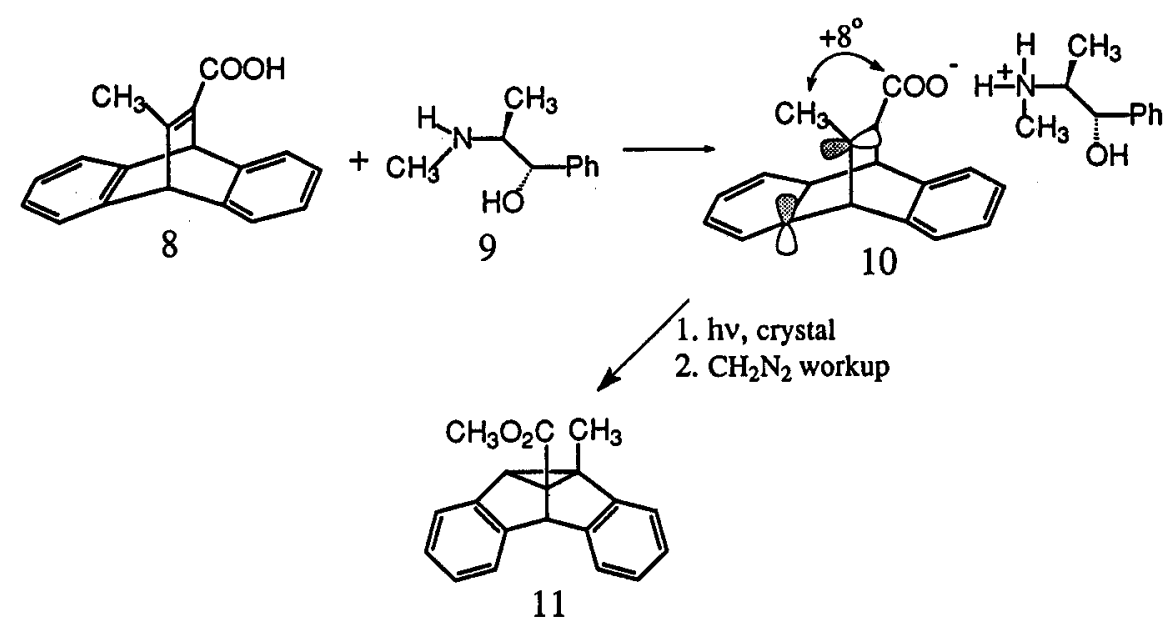

Since the absolute configuration of pseudoephedrine is known, the X-ray crystal structure of salt 10 establishes the absolute sign (and magnitude) of the $\mathrm{CH}_{3}-\mathrm{C}=\mathrm{C}-\mathrm{COO}$ dihedral angle, which was $+8^{\circ}$. In a separate experiment (the photoreaction of salt $\mathbf{1 0}$ is not a single crystal-to-single crystal process), the absolute configuration of photoproduct 11 was determined by transforming it into the corresponding amide of (S)-(-)- $\alpha$-methylbenzylamine and determining the crystal structure of this derivative (ref. 11). The correlated absolute configurations for reactant and product are those shown in Scheme 4. Once again, the results conform to the structure-reactivity relationship first elucidated for diester 3, that is, the direction of initial benzo-vinyl bridging (shown by the shaded orbitals in structure 10) is dictated by the sense of twisting about the vinyl double bond. This makes a total of five separate ethenoanthracene derivatives whose solid state photochemistry conforms to such a reactivity patter:l, and a sixth (not presented in this article in the interests of brevity) can be found in reference 13. It is likely, therefore, that this structure-reactivity relationship is general for compounds of this type. It is interesting to note that the structural features governing solid state reactivity in these cases are intranolecular in nature (orbital overlap and/or diminution of intramolecular steric interactions); crystal lattice effects seem to play a minor role other than to freeze the molecules in a single chiral conformation. A nice way of viewing the situation is to adopt the Bürgi-Dunitz approach (ref. 13) and consider these solid state conformers as molecules that are on their way to the transition state for the di- $\pi$-methane reaction, and that once initiated, there is an inherent tendency for these motions to be continued along the reaction coordinate rather than reversed.

\section{Absolute Configuration Correlations in The Norrish/Yang Type II Photoreaction}

As part of our ionic chiral auxiliary studies, we had occasion to investigate the solid state photochemistry of salt 12 (Scheme 5), formed by treating the corresponding achiral $\alpha$-adamantylacetophenone-pcarboxylic acid with (S)-(+)-prolinol (refs. 14,15). Crystals of salt 12 were dimorphic (plates and needles), and it is with the needle dimorph that we shall be concerned here. Irradiation of this dimorph (space group $\mathrm{P} 2,2,2$ ) in the solid state led to cyclobutanol derivative 13 as the major product following acidic workup and esterification with diazomethane. Yang photocyclization is favored in this case, since type II cleavage would lead to the very highly strained adamantene. Strikingly, photoproduct 13 was formed in $97 \%$ ee at a conversion of $87 \%$ (dextrorotatory enantiomer predominating); use of (R)-(-)prolinol led to (-)-13 in a similar $e e$. 


\section{Schemes}

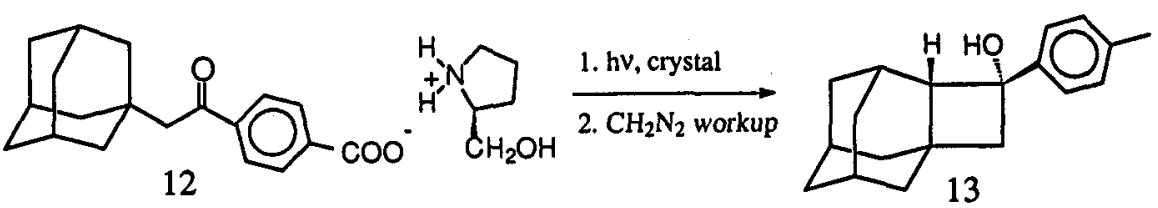

12

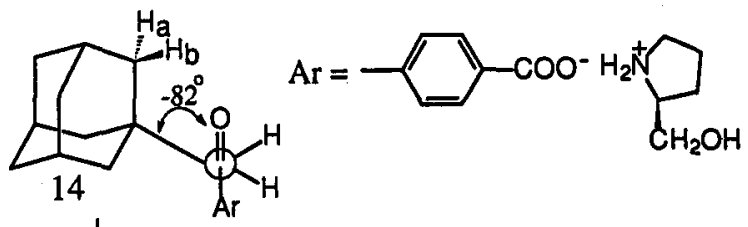

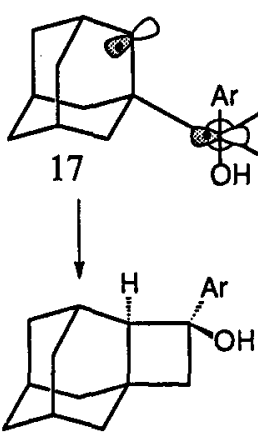

(S)-13

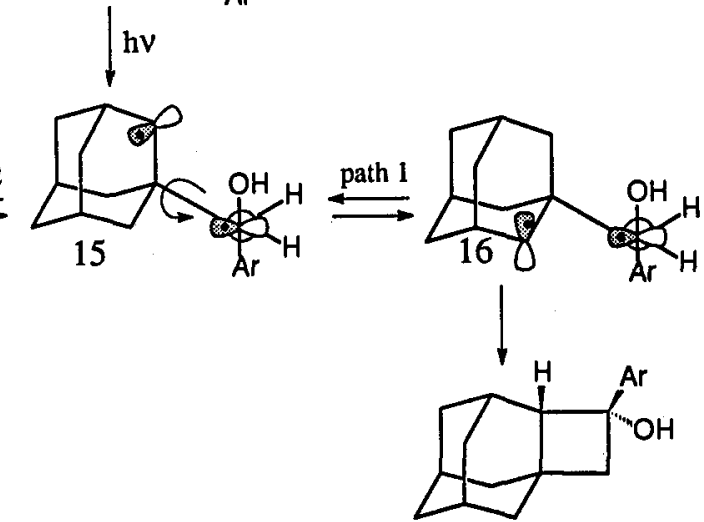

(R)-13

Since the absolute configuration of prolinol is known, the X-ray crystal structure gives us the absolute configuration of the photoreactive component of the starting salt, which we define by using the sign of the dihedral angle $O=C-C(\alpha)-C(\beta)$. For salt 12, this angle has a value of $-82^{\circ}$ (structure 14, Scheme 5). By good fortune, recrystallization of the crude reaction mixture from acetone deposited crystals that contained not only unreacted starting material, but the $(S)-(+)$-prolinol salt of the cyclobutanol photoproduct as well. A crystal structure of this complex was successfully determined (ref. 15), and this gave us the desired absolute configuration correlation, which was that the $-82^{\circ}$ starting material leads to the cyclobutanol that has the (R)-absolute configuration at the hydroxyl-bearing carbon atom.

We are now in a position to analyze the course of the solid state reaction. Initial hydrogen atom abstraction undoubtedly involves the nearest $\gamma$-hydrogen $\left(\mathrm{H}_{b}, 2.70 \AA\right)$, and this leads to 1,4hydroxybiradical 15, assumed for convenience to have the same conformation as the starting material. At this point, instead of closing directly by overlap of the shaded orbitals, a process that would lead to unfavorable steric interactions between the aryl group and the adamantane ring, biradical 15 leads to the less hindered photoproduct 13. This can happen in two ways: (1) by rotation of biradical 15 about the $C(\alpha)-C(\beta)$ bond to give biradical 16, which undergoes closure to the $(R)$-form of photoproduct 13, or (2) by rotation about the $\mathrm{C}(\alpha)-\mathrm{C}(\mathrm{OH}) \mathrm{Ph}$ bond to form biradical 17 , which could then close to the $(\mathrm{S})$ enantiomer of cyclobutanol 13. The absolute configuration correlation indicates that pathway 1 is followed, and this makes sense because it should clearly be easier for the spherical adamantane moiety to rotate about an axis in the solid state than for the aryl and hydroxyl groups to exchange positions, a process that would disrupt the strong ionic and hydrogen bond forces between the carboxylate anion and the prolinol cation.

A final important point concerns the enantioselectivity of the fluid phase photorearrangement of salt 12 . Irradiation of this material in chloroform led to no measurable optical activity in photoproduct 13. Similar results have been obtained for all ionic chiral auxiliary salts investigated. Evidently, in solution, the ions are not sufficiently well oriented or closely associated to lead to significantly different diastereomeric transition state free energies. 


\section{Asvmmetric Photocvclization of $m$-chlorophenyl- $N, N$-diisopropylglyoxalamide}

We close this paper with a brief account of some very recent work by Hashizume et al. on an absolute configuration correlation carried out on the solid state photochemical reaction of $m$-chlorophenyl- $N, N$ diisopropylglyoxylamide (18) (ref. 16). This achiral compound was found to crystallize spontaneously in the now familiar chiral space group $\mathrm{P} 22_{1} 2_{1}$, and substantial amounts of enantiomorphously homogeneous crystals of this material could be obtained by the seeding technique mentioned earlier. As shown in Scheme 6, irradiation of these crystals led to the $\beta$-lactam derivative 19 in what was reported to be $100 \%$ ee according to chiral HPLC (conversion unspecified). Interestingly, the $o$ - and $p$-chlorophenyl analogs of compound 18 were found to crystallize in achiral space groups, and their solid state photolysis afforded racemic $\beta$-lactams.

By taking advantage of the presence of chlorine as a heavy atom, the absolute structure of diketone 18 along with the absolute configuration of its photoproduct 19 were determined independently (nontopotactic reaction) by X-ray crystallographic Bijvoet analysis. In the correlated reactant/product pair, diketone 18 has a negative $\mathrm{O}(1)-\mathrm{C}(7)-\mathrm{C}(8)-\mathrm{N}(1)$ dihedral angle, and its photoproduct has the (S)-absolute configuration at $C(7)$, the newly formed stereogenic center. This correlation, shown by structures 20 and 21 (Scheme 6), is consistent with a mechanism in which the nearest $\gamma$-hydrogen, $H(9)$, is abstracted by $O(1)$ followed by closure of the intermediate 1,4-biradical with retention of configuration at $C(7)$. Thus, unlike the bimolecular deoxycholic acid/acetophenone system discussed earlier, there is no net rotation of the acyl group prior to radical coupling in the case of diketone 18. This is reasonable given the unfavorable steric consequences of inversion at $\mathrm{C}(7)$.
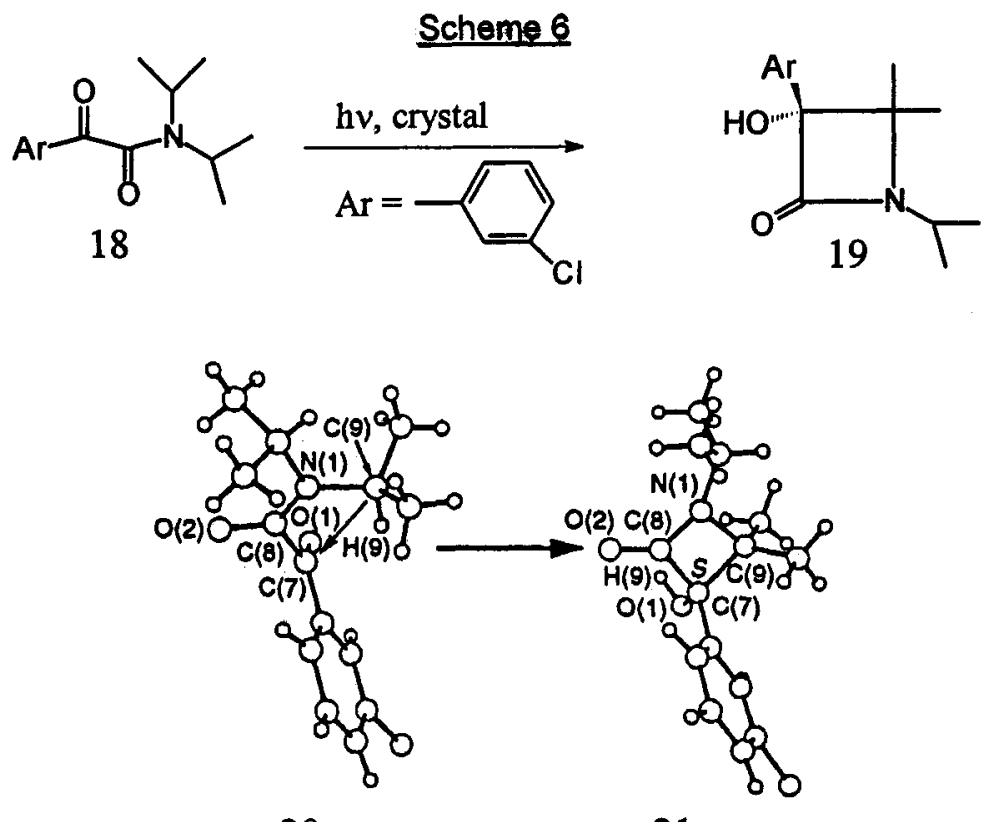

20

21

\section{SUMMARY}

The use of chiral crystalline environments to carry out asymmetric inductions in high optical yields is now established beyond question as a viable technique in organic photochemistry, and the results presented in this paper clearly show that absolute configuration correlation studies of such processes can tell us a great deal about the mechanisms involved as well as the subtle intra- and intermolecular forces that govern the motions of the molecules along the reaction coordinate. To date, only two types of photochemical reactions have been studied from this point of view-those initiated by hydrogen atom abstraction and those involving the di- $\pi$-methane rearrangement. The approach is not limited to these reactions, however, and current work in our laboratory is geared towards extending such studies to other common organic photochemical processes. 


\section{ACKNOWLEDGMENTS}

Acknowledgment is made to the donors of the Petroleum Research Fund, administered by the American Chemical Society, for partial support of this research. Financial support by the Natural Sciences and Engineering Research Council of Canada is also gratefully acknowledged.

\section{REFERENCES}

1. Y. Inoue. Chem. Rev. 92, 741 (1992).

2. (a) M. Vaida, R. Popovitz-Biro, L. Leiserowitz and M. Lahav. In Photochemistry in Organized and Constrained Media (V. Ramamurthy, ed), Chapter 6, VCH Publishers, New York (1991); (b) J.R. Scheffer and M. Garcia-Garibay. In Photochemistry on Solid Surfaces (M. Anpo and T. Matsuura, eds.), Chapter 9.3, Elsevier, New York (1989).

3. R. Popovitz-Biro, H.C. Chang, C.P. Tang, N.R. Shochet, M. Lahav and L. Leiserowitz. Pure and Appl. Chem. 52, 2693 (1980). See also reference 2a.

4. M. Garcia-Garibay, J.R. Scheffer, J. Trotter and F. Wireko. J. Am. Chem. Soc. 111, 4985 (1989).

5. Review. H.E. Zimmerman. In Molecular Rearrangements in Ground and Excited States (P. de Mayo, ed.), Chapter 16, Academic Press, New York (1980).

6. J.M. Bijvoet, A.F. Peerdeman and J.A. Van Bommel. Nature 168, 271 (1951).

7. T.Y. Fu, Z. Liu, J.R. Scheffer and J. Trotter. J. Am. Chem. Soc. 115, 12,202 (1993).

8. Z. Liu, Ph.D. Thesis, University of British Columbia, April, 1995.

9. J. Jacques, A. Collet and S.H. Wilen. Enantiomers, Racemates and Resolutions, pp. 14-18. Wiley, New York (1981).

10. J.N. Gamlin, R. Jones, M. Leibovitch, B. Patrick, J.R. Scheffer and J. Trotter. Acc. Chem. Res. 29, 203 (1996).

11. A.D. Gudmundadottir, J.R. Scheffer and J. Trotter. Tetrahedron Lett. 35, 1397 (1994).

12. J.R. Scheffer and J. Yang. In CRC Handbook of Onganic Photochemistry and Photobiology (W.M. Horspool and P-S. Song eds.), Chapter 16, CRC Press, Boca Raton, FL (1995).

13. H.B. Bürgi and J.D. Dunitz. Acc. Chem. Res. 16, 153 (1983)

14. R. Jones, J.R. Scheffer, J. Trotter and J. Yang. Tetrahedron Lett. 33, 5481 (1992).

15. R. Jones, J.R. Scheffer, J. Trotter and J. Yang. Acta Crystallogr. B50, 601 (1994).

16. D. Hahizume, H. Kogo, A. Sekine, Y. Ohashi, H. Miyamoto and F. Toda. J. Chem. Soc., Perkin Trans. 2, 61 (1996). 